\title{
卡式微柱凝胶法在猴血型鉴定中的应用
}

\author{
陈 松 ${ }^{1}$, 魏 晴 ${ }^{2}$, 李俊华 ${ }^{1}$, 向 莹 $^{1}$, 郭 晖 $^{1}$, 王婉瑜 ${ }^{3}$, 熊郁良 ${ }^{3}$, 陈 实 ${ }^{1}$, 陈 刚 $1^{*}$ \\ (1. 华中科技大学 同济医学院附属同济医院器官移植研究所, 湖北 武汉 430032; \\ 2. 华中科技大学 同济医学院附属同济医院血库, 湖北 武汉 430032; \\ 3. 中国科学院昆明动物研究所动物 毒素室, 云南 昆明 650223)
}

摘要: 灵长类动物的 $\mathrm{ABO}$ 血型抗原都表达在组织器官内, 而不是在红细胞上, 这给灵长类动物血型的鉴定 带来很大的困难。为找到更加简捷、准确鉴定灵长类动物类人ABO血型的方法, 采用近年来临床上广泛应用的卡 式微柱凝胶正、反定型法对34只猕猴和16只食蟹猴的血型进行了鉴定, 并与肾组织免疫组化法的检测结果进行比 较。结果显示: 卡式微柱凝胶正定型法的检测结果中无一例为阳性结果; 血浆中的纤维蛋白原和人一猴种属间非 特异性抗体都会对卡式微柱凝胶反定型法的部分检测结果产生干扰; 采用经正常人 $\mathrm{O}$ 型红细胞吸附处理后的清亮 血清, 卡式微柱凝胶反定型法的检测结果明确, 与免疫组化法判定结果一致。由此得出: 卡式微柱凝胶反定型法 可以用于灵长类动物血型的鉴定, 其主要干扰因素为血浆内的纤维蛋白原和人一猴种属间非特异性抗体, 在采用 清亮血清及经正常人 $\mathrm{O}$ 型红细胞吸附处理后能消除其干扰。

关键词: 卡式微柱凝胶法; ABO 血型; 猴

中图分类号：Q461；Q592.1 文献标识码：A 文章编号：0254-5853-(2008)03-0318-07

\section{Evaluation of the Gel System for ABO Typing of Monkeys}

\author{
CHEN Song ${ }^{1}$, WEI Qing ${ }^{2}$, LI Jun-hua ${ }^{1}$, XIANG Ying ${ }^{1}$, GUO Hui ${ }^{1}$, WANG Wan-yu ${ }^{3}$, \\ XIONG Yu-liang ${ }^{3}$, CHEN Shi ${ }^{1}$, CHEN Gang ${ }^{1, *}$ \\ (1. Institute of Organ Transplantation, Tongij Hospital, Tongji Medical College, Huazhong University of Science and Technology, Wuhan 430030, China; \\ 2. Blood Bank of Tongij Hospital, Tongji Medical College, Huazhong University of Science and Technology, Wuhan 430030, China; \\ 3. Kunming Institute of Zoology, the Chinese Academy of Sciences, Kunming 650223, China)
}

\begin{abstract}
Monkeys do not express ABO antigens on red blood cells (RBCs), but on tissues, which is an obstacle in ABO typing. In this study we describe a simple and efficient method to type monkeys blood. Rhesus monkeys $(n=34)$ and cynomolgus monkeys $(n=16)$ were typed by the direct and reverse gel system, which has been widely used in clinics in recent years. Based on the results of immunohistochemical staining, we evaluated the feasibility and the interference factors of the gel system to type monkeys. The results revealed that the direct gel system didn't have a positive report, the fibrinogen and non-specific anti-human antibodies in monkey blood interfered with the reverse gel system in some samples. In accordance with the results of immunohistochemical staining, using clear sera, which were pre-absorbed on normal human type O RBCs, the reverse gel system gave an accurate blood type determination for monkeys. We concluded that the reverse gel system could be used for ABO typing of monkeys, and the fibrinogen and non-specific anti-human antibodies were the major interference factors, which could be eliminated by using clear and pre-absorbed sera.
\end{abstract}

Key words: Gel system; ABO blood group; Monkey

近似于人类，灵长类动物也有 $\mathrm{ABO}$ 血型系统。 但和人类及少部分灵长类动物（如类人猿）不同， 绝大部分灵长类动物 (如猕猴、食蟹猴、狒狒等) 的外周血红细胞上并不表达 $\mathrm{AB}$ 抗原, 或是非常弱
（Doxiadis et al, 1998），而在腺体、上皮、血管内 皮系统和其他组织内均有广泛的 A 或/和 B 抗原表 达 (Oriol et al,1984; Socha et al,1987; Oriol et al,1993; Rydberg et al, 2001), 循环系统中也有抗 A 或/和抗

收稿日期：2008-04-25; 接受日期：2008-05-12

基金项目：国家自然科学基金资助项目（C03020505）

*通讯作者 (Corresponding author), Tel: 027-83662655, E-mail: gchen@tjh.tjmu.edu.cn 
$\mathrm{B}$ 抗体的存在。这些血型抗体与移植器官上表达的 $\mathrm{AB}$ 抗原结合同样可以造成类似于人类 $\mathrm{ABO}$ 血型不 合的同种异体器官移植排斥反应 (Oriol et al, 1984; Socha et al,1987; Cooper et al,1989)。故准确的检测 灵长类动物类人 $\mathrm{ABO}$ 血型, 对于某些相关科研领 域极为重要, 对更加全面的了解灵长类动物的血型 系统特点也有重要的意义。

由于灵长类动物的 $\mathrm{ABO}$ 血型抗原并不表达在 外周血红细胞上, 所以其血型鉴定一直较难。鉴定 灵长类动物血型的常用方法一般是传统试管法抗 球蛋白试验(tube anti-globulin technique，TAT)和毛 果云香碱用药后唾液的 $\mathrm{AB}$ 抗原鉴定 (Cooper et al,1989; Moor et al,1964)。这两种方法均为手工操 作, 存在不易标准化、人为因素大、影响因素多的 缺点, 容易出现假阳性或者假阴性, 造成血型不能 明确判定或判定错误。而检测灵长类动物血型最可 靠的方法是组织切片免疫组织化学染色法, 但是由 于其有创性, 标本不易获得, 故一般只用于回顾验 证性研究。

卡式微柱凝胶法(以下称卡式法)是法国 Yves Lapierre 在 1986 年首先发明的一项免疫学检测新 技术, 是微柱凝胶技术与以往所有血型鉴定技术相 结合的产物。其敏感性高, 可用于临床困难血型的 鉴定。1994 年此项技术获得了美国 FDA 的认可。 经过不断改进和临床大量应用, 在许多发达国家此 项技术已作为常规的红细胞血型血清学检测技术 应用于临床 ( $\mathrm{Li}$ et al,1999; Titlestd et al, 1997; Langston et al,1999; Cate et al,1999)，但目前尚未见 将其应用于灵长类动物血型鉴定的报道。本研究以 灵长类肾组织血型免疫组化检测结果为可靠的参 照标准, 探讨卡式法用于猕猴和食蟹猴血型鉴定的 效果, 并研究其干扰因素和消除方法。

\section{1 材料与方法}

\section{1 实验动物及样本采集}

健康猕猴 34 只和食蟹猴 16 只均来自中国科学 院昆明动物研究所实验动物中心和广州蓝岛生物 技术有限公司华南灵长类研究开发中心, 狝猴体重 约 5-10 kg, 食蟹猴体重约 3-5kg, 均为雄性。

血浆及血清标本采集: 用肝素钠抗凝管采血. 3 $\mathrm{mL}$, 用 $3800 \mathrm{r} / \mathrm{min}$ 离心 (德国 Eppendorf 离心机, 型号：5810）10 min 后，获得新鲜血浆; 用促凝管 采血 $3 \mathrm{~mL}$, 室温静置 $60 \mathrm{~min}$ 后, $4^{\circ} \mathrm{C}$ 冰箱内静置过
夜; 待清亮血清析出后, $3800 \mathrm{r} / \mathrm{min}$ 离心 $10 \mathrm{~min}$, 获 得新鲜血清。

血清吸附处理: 为去除猴血清中存在的抗人非 特异性抗体, 取 $200 \mu \mathrm{L}$ 新鲜血清, 与 $200 \mu \mathrm{L}$ 浓缩 正常人 $\mathrm{O}$ 型红细胞 (上海血液生物医药有限责任公 司）混匀后, $37^{\circ} \mathrm{C}$ 捊育 $30 \mathrm{~min}, 1372 \mathrm{r} / \mathrm{min}$ 离心 (德 国 Eppendorf 离心机, 型号: 5417R）10min 后取上 清, 得吸附后血清。

\section{2 玻片凝集法鉴定猴血型}

玻片凝集法是测定人 $\mathrm{ABO}$ 血型的常规方法。 其原理是检测外周血红细胞上表达的 $\mathrm{AB}$ 抗原类 型。为了验证猴红细胞上是否表达 $\mathrm{AB}$ 血型抗原, 本研究采用经免疫组化法已经明确血型的 3 只猴 (07R09 AB 型, 07cy02 A 型, 07R03 B 型) 作为 待测猴, 并用已知血型的正常人作为阳性对照。

玻片凝集法步骤：在玻片上分别滴上一滴抗 A 和一滴抗 B 血清 (长春生物制品研究所), 在每一 滴血清上再加一滴待测猴或人全血, 轻轻摇动, 使 血清和红细胞混匀, 在 10-30 min 内观察有无凝集 现象。

\section{3 卡式法鉴定猴血型}

DiaMed 卡式微柱凝胶系统由 6 根微管组成, 微管内装经缓冲调节的由 Sephadex 组成的中性凝 胶, 凝胶内预混有一定浓度的抗人球蛋白(AHG)溶 液。该微柱凝胶系统利用微柱中填充物(即凝胶)的 空间位阻, 在一定离心力的作用下, 只允许单个红 细胞通过, 所以有抗体结合的凝集红细胞由于体积 增大而无法通过微柱, 而留在微柱上端（判为阳 性); 没有抗体结合的单个红细胞则可以通过微柱 沉至柱底 (判为阴性) (图 1)。

卡式正定型法：在 $\mathrm{ABO} / \mathrm{D}$ 血型凝胶卡（瑞士 DiaMed 公司）正定型侧的 A、B 及 D 管侧（已有 抗 A、抗 B 及抗 $\mathrm{Rh}$ 标准液）分别加入 $10 \mu \mathrm{L} 5 \%$ 的 待测猴红细胞悬液, DiaMed 卡式专用离心机（瑞 士, 型号: DiaMed-ID 12 S II ) 1030r/min 离心 10 min 后, 判定结果 (图 1a)。A 管内含有抗 A 抗体标准 液, 若出现凝集红细胞, 显示阳性结果, 则证实待 测猴红细胞上存在 $\mathrm{A}$ 抗原, 血型判为 $\mathrm{A}$ 型; 同理, 若 B 管出现阳性结果, 则证实待测猴红细胞上存在 $B$ 抗原, 血型判为 $B$ 型; 若 $A 、 B$ 管均为阳性或均 为阴性, 则证实待测猴红细胞上同时存在或不存在 $A 、 B$ 抗原，血型分别判为 $A B$ 型或 $O$ 型。

卡式反定型法: 在 $A B O / D$ 血型凝胶卡反定型 
侧的 ctl、 $\mathrm{A}_{1}$ 及 $\mathrm{B}$ 管分别加入 $50 \mu \mathrm{L}$ O、 $\mathrm{A}_{1} 、 \mathrm{~B}$ 型标 准反定型细胞 (上海血液生物医药有限公司) 和 50 $\mu \mathrm{L}$ 待测猴血浆或血清, DiaMed 卡式专用离心机 $1030 \mathrm{r} / \mathrm{min}$ 离心 $10 \mathrm{~min}$ 后, 判定结果 (图 1b)。 $\mathrm{A}_{1}$ 管内含有 $\mathrm{A}_{1}$ 型标准红细胞, 若待测猴血清内存在 抗 $\mathrm{A}$ 抗体, 则会出现凝集红细胞, 显示阳性结果, 则判为 $\mathrm{B}$ 型; 同理, 若 $\mathrm{B}$ 管出现阳性结果, 则证实 待测猴血清内存在抗 $B$ 抗体, 血型判为 $A$ 型; $A$ 、 $\mathrm{B}$ 管均为阳性或均为阴性, 则证实待测猴血清内同 时存在或不存在抗 $A$ 和抗 $B$ 抗体, 血型分别判为 $O$ 型或 $A B$ 型。由于卡式法的高灵敏性, 容易出现假 阳性, 故设置了 $\mathrm{ctl}$ 对照管。ctl 管内含有 $\mathrm{O}$ 型标准 红细胞, 因 $\mathrm{O}$ 型标准红细胞上没有 $\mathrm{AB}$ 抗原的表达, 故无论待测猴为何种血型, 均不应该产生凝集红细 胞, 若出现了阳性结果, 则说明有干扰因素存在, 判为假阳性。因血浆与血清相比含较多纤维蛋白 原, 为研究标本中纤维蛋白原及抗人非特异性抗体 对卡式反定型法的干扰作用, 检测分以下 3 组: 1) 新鲜血浆；2）新鲜血清；3）吸附后血清。

\section{4 猴肾脏组织的免疫组织化学染色}

猴肾脏标本经 $10 \%$ 中性福尔马林固定 48h 后, 经常规脱水、浸蜡、制成 3-4 $\mathrm{mm}$ 的切片 (用经 APES 处理过的载玻片粘片) 、烤片 $30 \mathrm{~min}$ 后, 室温放置 备用。

常规免疫酶法组织化学染色步骤: 石蜡切片经 常规步骤透明, 水洗 $4 \mathrm{~min}, 3 \% \mathrm{H}_{2} \mathrm{O}_{2}$ 处理 $10 \mathrm{~min}$,
$10 \%$ 正常羊血清 $37^{\circ} \mathrm{C}$ 封闭 $60 \mathrm{~min}$, 鼠抗 $\mathrm{A}$ 或抗 $B$ 单克隆 $\operatorname{IgM}$ (长春生物制品研究所) $4^{\circ} \mathrm{C}$ 孵育过夜, 生物素化羊抗鼠 IgM 二抗室温孵育 $20 \mathrm{~min}$, 辣根酶 标记链酶卵白素工作液（北京中杉 SP 试剂盒）室 温捊育 $20 \mathrm{~min}, \mathrm{DAB}$ 显色 (北京中杉 DAB 试剂盒), 出现黄褐色着色则判为阳性。

以上步骤中, 除了 $10 \%$ 正常羊血清封闭后直接 滴加一抗, 其他步骤间均需 PBS 洗 $3 \mathrm{~min} \times 3$ 次。DAB 显色后常规苏木素染色 $1 \mathrm{~min}$, 盐酸酒精分化 $10 \mathrm{~s}$, 逐级酒精脱水、二甲苯透明后, 中性树胶封片。

\section{2 结 果}

\section{1 常规玻片凝集法的检测结果}

用常规玻片凝集法对已知为 $A 、 B 、 A B$ 血型的 猴进行血型检测, 无一例出现红细胞凝集反应, 而 人阳性对照组均出现相应的典型红细胞凝集反应 (图 2)。
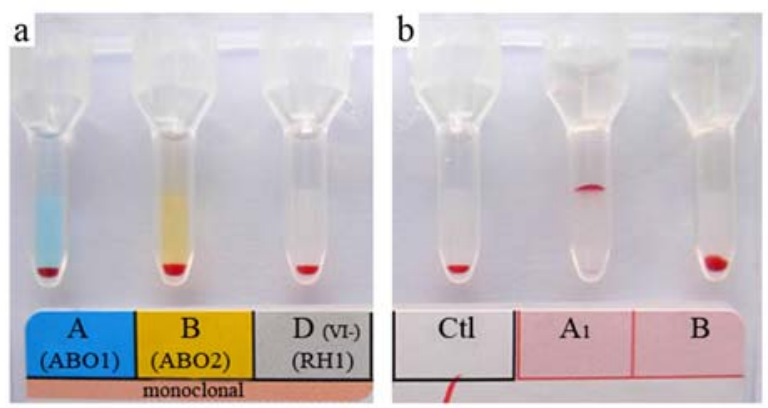

图 1 卡式微柱凝胶法图解

Fig. 1 The micro-column gel system a: 正定型侧(The direct site); b: 反定型侧(The reverse site)。

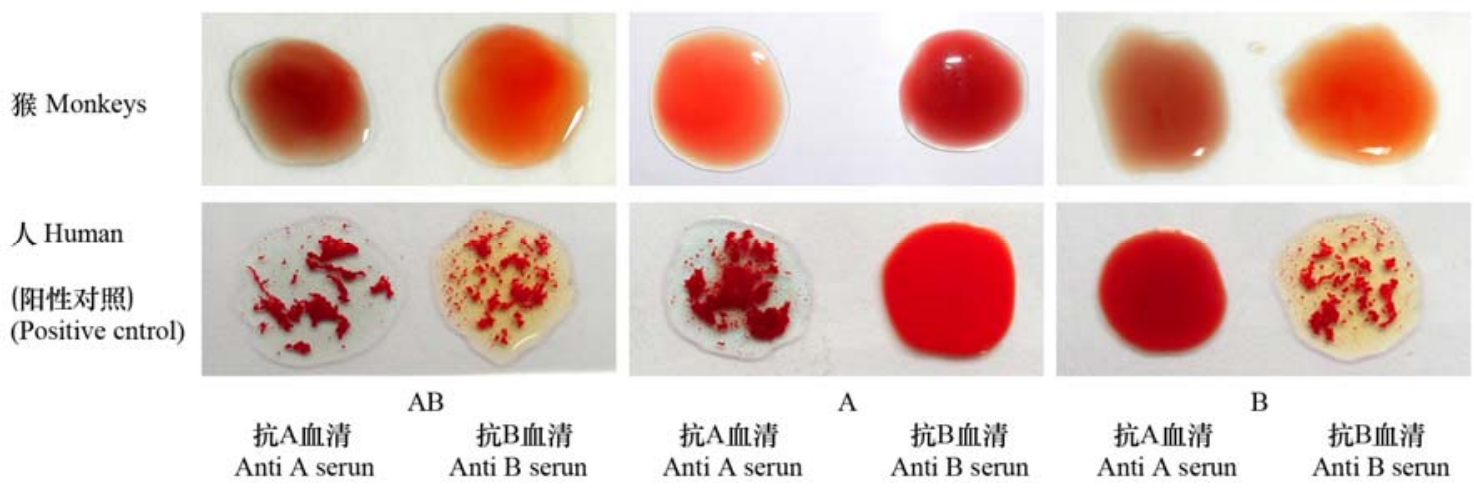

图 2 玻片凝集法检测结果

Fig. 2 The results of slide agglutination 猴的 3 种血型类别均未出现阳性凝集; 人阳性对照均出现典型的阳性凝集。

These is no positive agglutination in 3 monkeys with different blood types, but typical agglutination in the human positive control. 


\section{2 卡式正定型法的检测结果}

用卡式正定型法对 34 例猕猴和 16 例食蟹猴的 血型进行鉴定, 无一例出现阳性结果, 图 3 显示 3 只代表性猴的血型用免疫组化法检测分别为 $A B$ 、

$A 、 B ，$ 但卡式正定型法结果均为阴性。

\section{3 卡式反定型法的检测结果}

采用血浆标本对卡式反定型法的结果干扰较 大, 如图 4a 所示, 绝大多数检测仅出现部分红细 胞通过微柱沉至柱底, 血型判定结果不明确。

采用清亮的血清标本 (不含或仅含少量纤维蛋 白原)，则在很大程度上消除了这种干扰（图 4b), 但仍存在少许拖尾现象。因干扰明显减少, 34 例狝 猴采用血清标本检测后能准确判定血型 (与组化结 果对比), 但 16 例食蟹猴采用血清标本检测后仍出 现不同程度的干扰现象 (图 5a)。采用正常人 O 型 红细胞吸附后的血清标本较好地消除了干扰（图 $5 b)$ 。

\section{4 猴类人 $\mathrm{ABO}$ 血型分布情况}

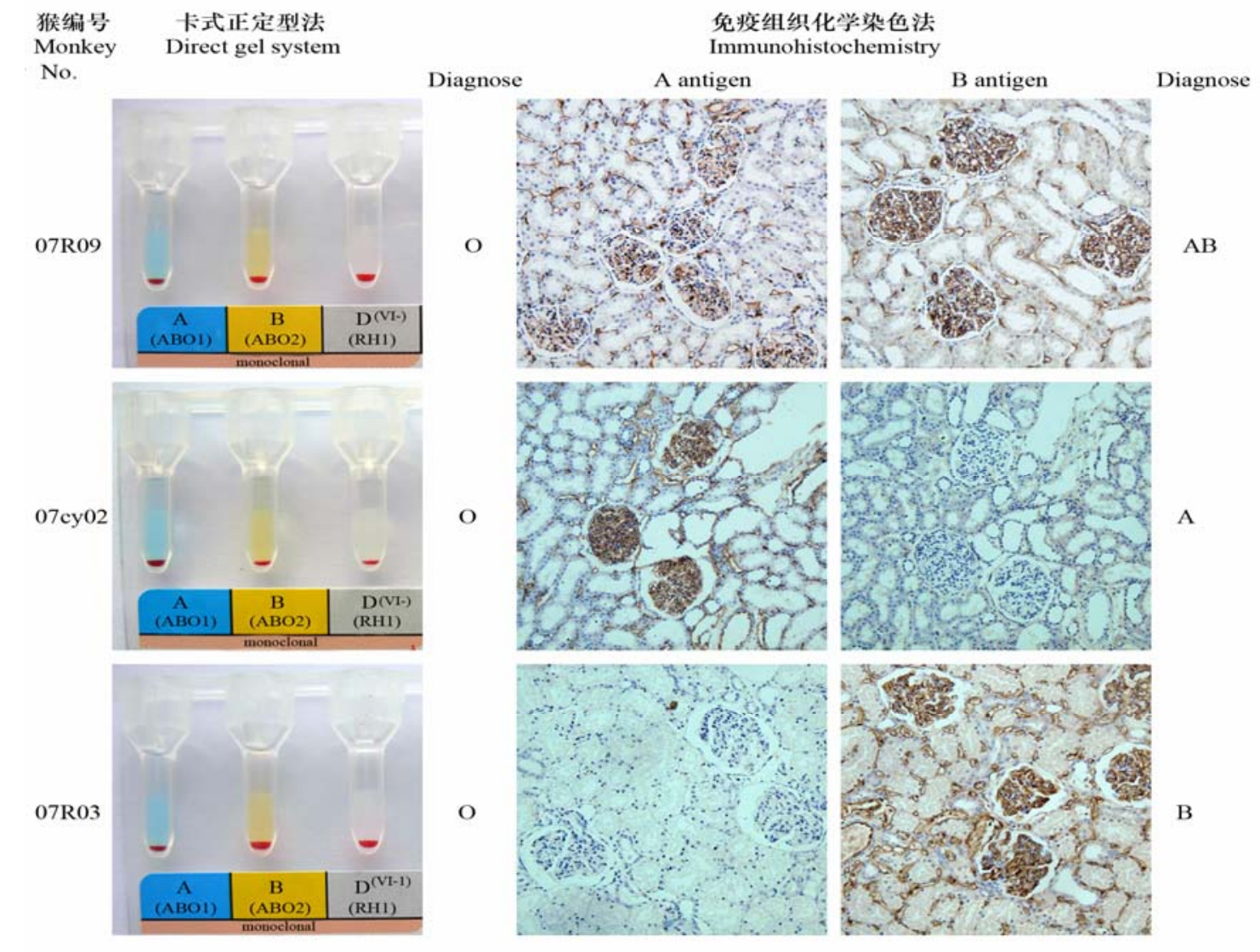

图 3 卡式正定型法对猴血型的判定

Fig. 3 The results of the direct gel typing system
经消除干扰的卡式反定型法和肾组织免疫组 化检测结果对比, 猴血型判定的符合率达 100\%（图 6 , 表 1)。所检测 34 例猕猴中绝大多数为 B 型 (26 例), $\mathrm{AB}$ 型其次 (5 例), $\mathrm{A}$ 型及 $\mathrm{O}$ 型少见 (分别 为 1 例及 2 例); 16 例食蟹猴中也是以 $\mathrm{B}$ 型居多 (8 例), 其次为 $\mathrm{A}$ 型和 $\mathrm{AB}$ 型 (各 4 例), $\mathrm{O}$ 型末见 (表 1)。

\section{3 讨 论}

传统的玻片凝集法和卡式正定型法的检测原 理都是建立在红细胞表达 $\mathrm{A}$ 或/和 $\mathrm{B}$ 抗原的基础上。 虽然在临床应用上，玻片凝集法已基本被淘汰，但 是本实验利用其比较直观的特点, 并与卡式正定型 法进一步检测相结合, 证实猕猴或食蟹猴红细胞上 不表达 A 或/和 B 抗原，故玻片凝集法和卡式正定 型法均不适用于猕猴或食蟹猴血型的检测。

在本研究中, 用新鲜血浆进行卡式反定型试 验, 均出现了明显的假阳性和干扰, 可能主要为血 
a
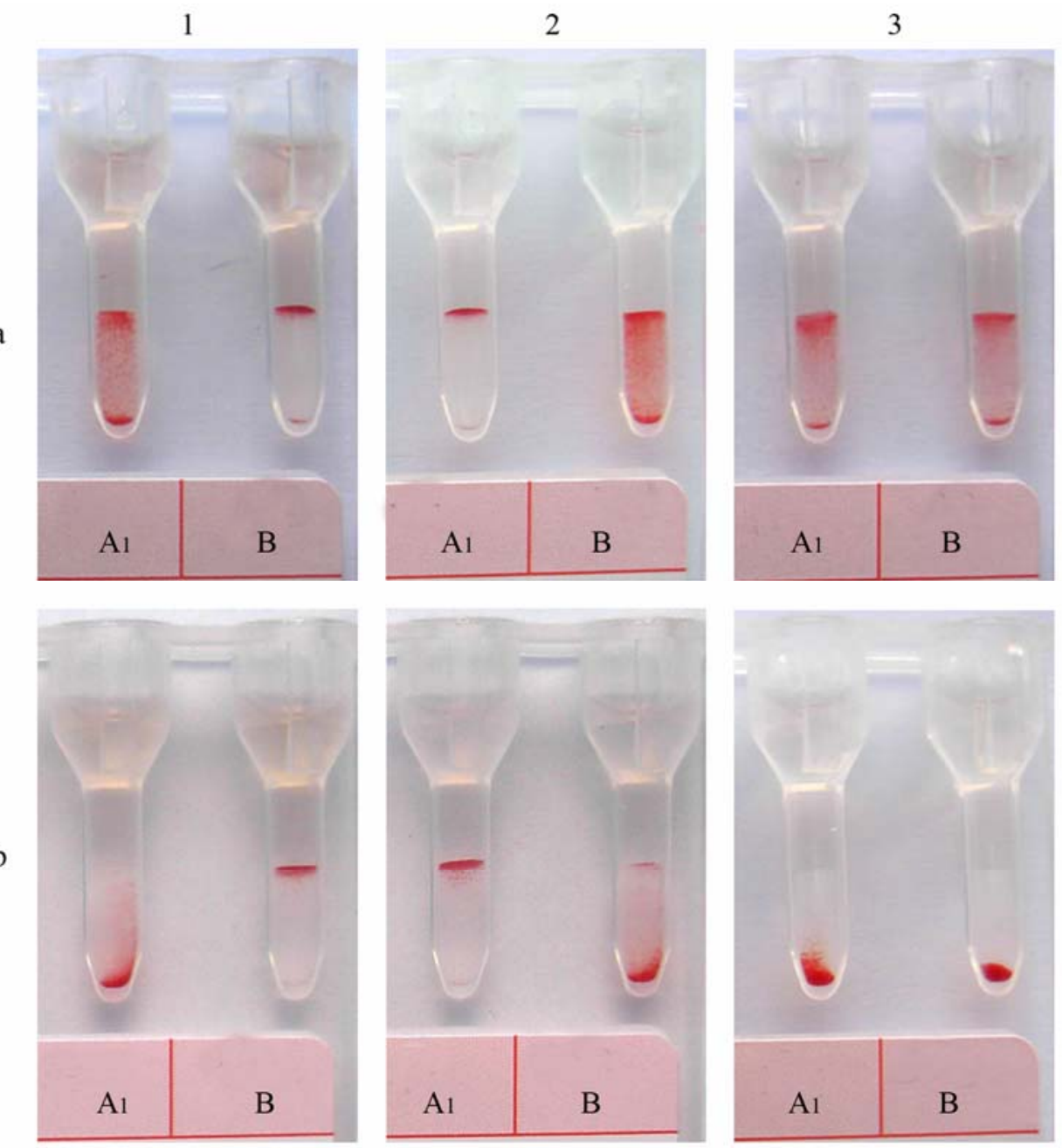

图 4 采用猴血浆和血清行卡式反定型法的结果比较

Fig. 4 The comparison of results using plasma and serum in the reverse gel typing system a: 新鲜血浆(Fresh plasma); b: 新鲜血清(Fresh serum)。1: 猴 07cy09(Monkey 07cy09); 2: 猴 07R05(Monkey 07R05); 3: 猴 07cy10(Monkey 07cy10)。
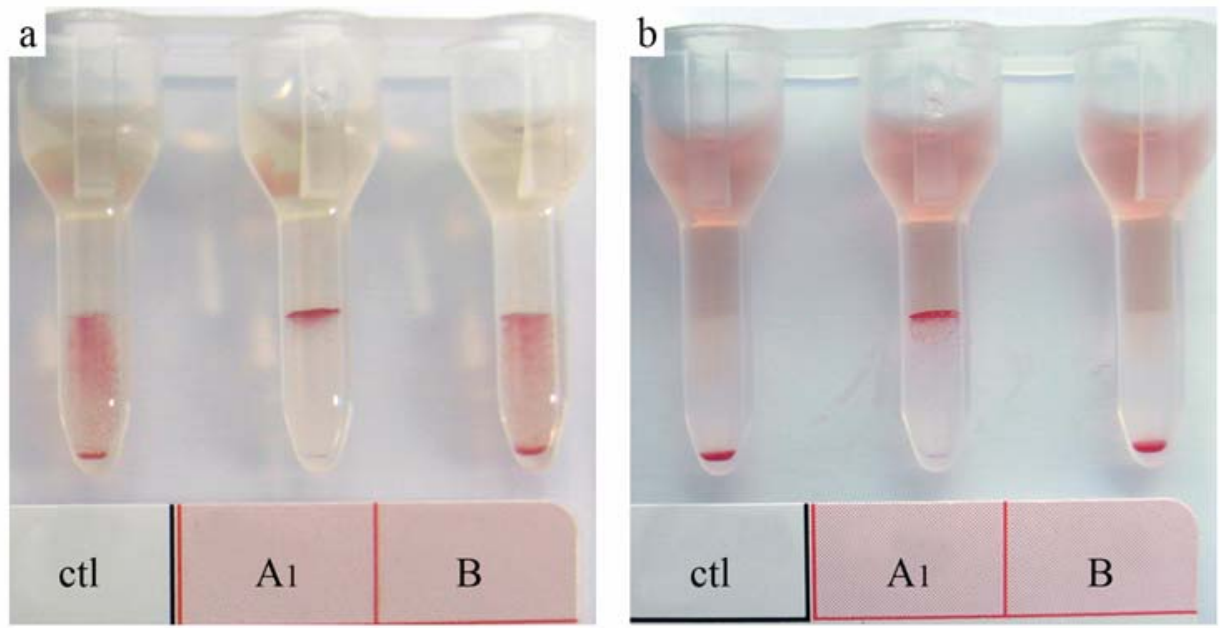

图 5 采用猴血清和吸附后血清行卡式反定型法的结果比较（猴 07cy04）

Fig. 5 The comparison of results using serum and pre-absorbed serum in the reverse gel typing system (monkey 07cy04) a: 新鲜血清 (Serum); b: 吸附后血清 (Pre-absorbed serum)。 


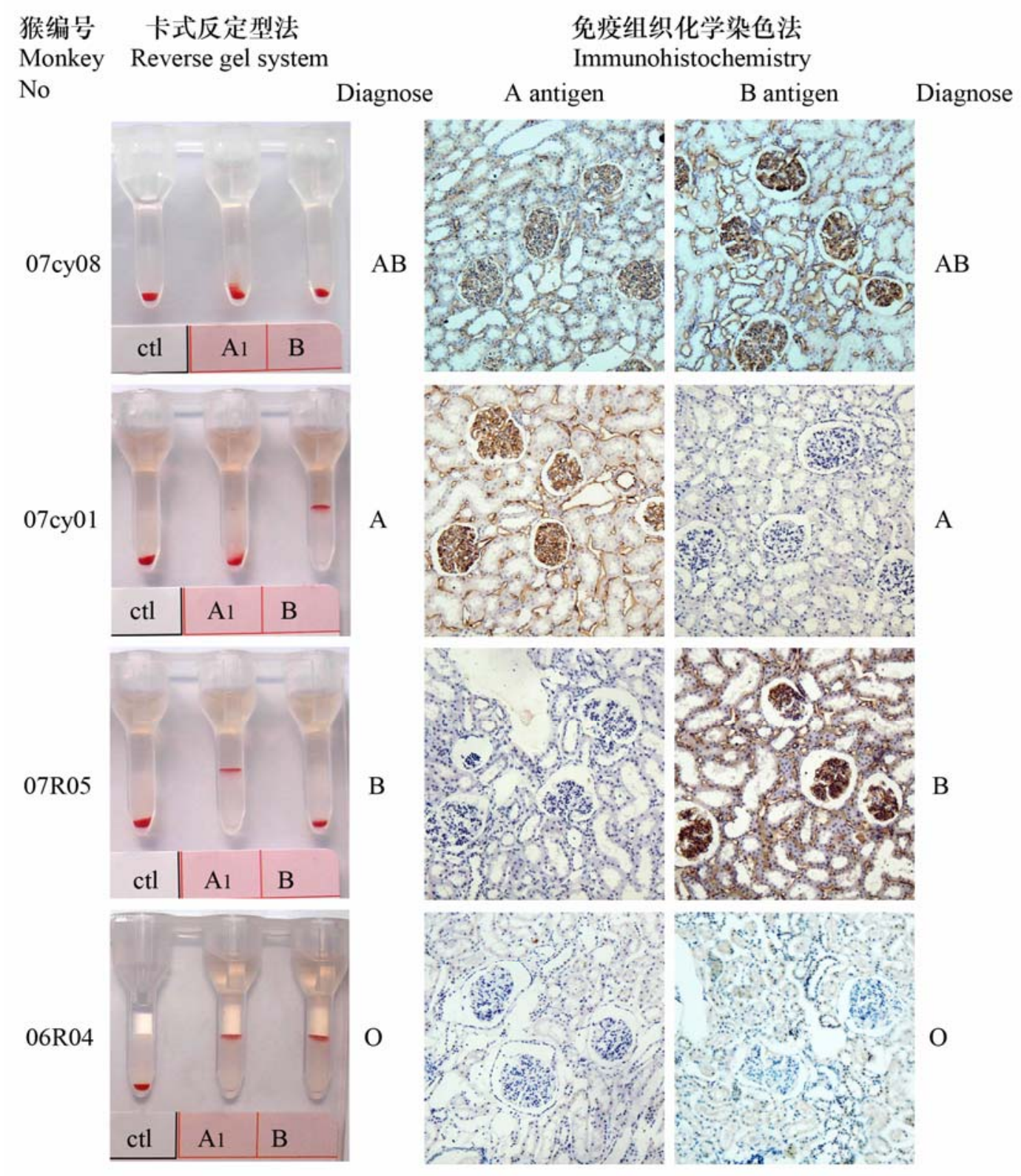

图 6 卡式反定型法对猴血型的判定

Fig. 6 The results of the reverse gel typing system

表 1 猴血型判定结果

Tab.1 The final results of ABO typing of monkeys

\begin{tabular}{|c|c|c|c|c|c|c|c|c|}
\hline \multirow{2}{*}{ 猴种类 Species } & \multicolumn{4}{|c|}{ 卡式反定型法 Reverse gel system } & \multicolumn{4}{|c|}{ 免疫组织化学染色法 Immunohistochemistry } \\
\hline & A & $\mathrm{B}$ & $\mathrm{AB}$ & $\mathrm{O}$ & A & $\mathrm{B}$ & $\mathrm{AB}$ & $\mathrm{O}$ \\
\hline 猕猴 Rhesus monkeys (n=34) & 1 & 26 & 5 & 2 & 1 & 26 & 5 & 2 \\
\hline 食蟹猴 Cynomolgus monkeys (n=16) & 4 & 8 & 4 & 0 & 4 & 8 & 4 & 0 \\
\hline
\end{tabular}

浆中的纤维蛋白原所致, 因新鲜血浆中含有较丰富 的纤维蛋白原, 在检测过程中析出的纤维蛋白可以 形成网状结构影响部分红细胞通过凝胶柱。这种现 象以往在临床标本的检测中也有类似报道 (Zhou et al, 2005; American Association of Blood Banks, 2002; Wu et al, 2006)。采用新鲜血清在很大程度上避免了 纤维蛋白原的干扰, 对狝猴已经能准确的判定血型 结果, 但在食蟹猴血型的检测中仍存在不同程度的 
干扰现象, 可能为血清中存在的抗人非特异性抗体 所致, 采用正常人 $\mathrm{O}$ 型红细胞吸附处理后能有效消 除干扰, 证实了以上假设。

采用经正常人 $O$ 型红细胞吸附后的清亮血清, 用卡式反定型法对 34 例猕猴和 16 例食蟹猴进行血. 型检测, 判定结果均很明确, 且与免疫组织化学染 色法的判定结果完全一致。与传统试管法抗球蛋白 试验、毛果云香碱用药后唾液的 $\mathrm{AB}$ 抗原检测法比 较, 卡式法具有以下优势: (1) 采用标准化定量操 作, 最大限度减少操作人员的随意性, 重复性好;

（2）采用凝胶分子篎技术，将凝集红细胞与游离 红细胞分离开, 使结果一目了然, 避免了镜下的主 观影响; (3) 操作简便, 省去繁琐的洗涤过程, 不 再担心抗人球蛋白抗体被中和而产生假阴性;（4） 结果便于保存, 一般置室温可保存数月至 1 年, 还 可进行拍照摄像提供永久记录, 以备发生血型鉴定

\section{参考文献:}

American Association of Blood Banks. 2002. Technical Manual 14th Ed. [M]. Bethesda:AABB Press, 687-688.

Busch J, Specht S, Ezzelarab M, Cooper DKC. 2006. Buccal mucosal cell immunohistochemistry: a simple method of determining the $\mathrm{ABH}$ phenotype of baboons, monkeys, and pigs[J]. Xenotransplantation, 13: 63-68

Cate JC, Reilly N. 1999. Evaluation and implementation of the gel test for indirect antiglobulin testing in a community hospital laboratory [J]. Arch Pat hol Lab Med, 123(8):693.

Cooper DK, Human PA, Rose AG, Rees J, Keraan M, Reichart B, Du Toit E, Oriol R. 1989. The role of $\mathrm{ABO}$ blood group compatibility in heart transplantation between closely related animal species. An experimental study using the vervet monkey to baboon cardiac xenograft model[J]. J Thorac Cardiovasc Surg, 97: 447.

Doxiadis GG, Otting N, Antunes SG, de Groot NG, Harvey M, Doxiadis II, Jonker M, Bontrop RE. 1998. Characterization of the ABO blood group genes in macaques: evidence for convergent evolution[J]. Tissue Antigens, 51: 321.

Langston MM, Procter JL, Cipolone KM, Stroncek DF. 1999. Evaluation of the gel system for ABO grouping and D typing [J]. Transfusion, 39(3): 300.

LI Y, Yang GZ. 1999. The Application and Laboratorial Technique of the Human Red Blood Cell Typology[M]. Beijing: China Publ House Science and Technology, 276-278. [李 勇, 杨贵贞. 1999. 人类红细 胞血型学使用理论与实验技术. 北京: 中国科学技术出版社, 276-278.]

Moor JJ, Wiener AS, Gordon EB. 1964. Blood groups of apes and monkeys.
差错时查找原始资料;（5）塑料卡易于销毁。Busch et al（2006）报道，可以用棉签蘸拭法获取猴口腔 粘膜上皮, 涂片后, 经 $\mathrm{AB}$ 抗原的免疫苂光染色也 可以比较方便、准确的获知猴的类人 $\mathrm{ABO}$ 血型。 但与卡式法相比, 该法仍然存在人为因素大、操作 步骤多、不易标准化等缺点。

另外, 本实验还发现狝猴的血型绝大多数为 B 型, 其他血型较少见, 食蟹猴也是以 $\mathrm{B}$ 型为主, 但 $\mathrm{A}$ 及 $\mathrm{AB}$ 型也较常见, $\mathrm{O}$ 型罕见, 这种血型分布趋 势与国外报道相似 (Socha et al, 1987)。

综上所述, 采用经人正常 $\mathrm{O}$ 型红细胞吸附处理 后的清亮血清作为样本，卡式微柱凝胶反定型法可 以准确的判定狝猴或食蟹猴的血型, 是个更为简 单、快捷的方法, 推荐为灵长类动物血型检测的常 规方法。

I. The ABO blood groups in baboons[J]. Transfusion, 4: 92.

Oriol R, Cooper JE, Davies DR, Keeling PW. 1984. ABH antigens in vascular endothelium and some epithelial tissues of baboons[J]. Lab Invest, 50: 514.

Oriol R, Ye Y, Koren E, Cooper DK. 1993. Carbohydrate antigens of pig tissues reacting with human natural antibodies as potential targets for hyperacute rejection in pig-to-man organ xenotransplantation[J]. Transplantation, 56: 1433.

Rydberg L, Mölne J, Strokan V, Svalander CT, Breimer ME. 2001. Histo-blood group A antigen expression in pig kidneys--Implication for ABO incompatible pig-to-human xenotransplantation[J]. Scand J Urol Nephrol, 35: 54.

Socha WW, Marboe CC, Michler RE, Rose EA, Moor-Jankowski J. 1987. Primate animal model for the study of ABO incompatibility in organ transplantation[J]. Transplant Proc, 19: 4448.

Titlestd K, Georgsen J, Andersen H, Kristensen T. 1997. Detection of irregular red cell antibodies: more than 3 years of experience with a gel technique and pooled screening cells [J]. Vox Sang, 73(4): 246.

Wu YJ, Zhu XH, Liu YH, Li CJ, Chen BC, Wu Y. 2006. Application of micro-column gel anti-globulin cards in blood group serological detecting[J]. Chn J Lab Diag, 10(2):174. [吴远军, 朱学海, 刘彦慧, 李重江, 陈宝婵, 吴 勇. 2006. 国产微柱凝胶抗球蛋白卡在血型 血清学试验中的应用. 中国实验诊断学, 10(2):174.]

Zhou W, Ding QL, Wang XF. 2005. The limitation of the gel card in the use of the blood type determination[J]. J Diag, 4(5):410. [邹 纬, 丁秋兰, 王学锋. 2005. 血型卡在血型鉴定中的局限性. 诊断学理论与实践, 4(5): 410.] 